\title{
Study Design of the Graft Patency After FFR-Guided Versus Angiography-Guided CABG Trial (GRAFFITI)
}

\author{
Gabor G. Toth ${ }^{1,2} \cdot$ Bernard De Bruyne $^{2} \cdot$ Petr Kala $^{3} \cdot$ Flavio L. Ribichini ${ }^{4} \cdot$ Filip Casselman $^{2} \cdot$ Ruben Ramos $^{5}$. \\ Zsolt Piroth $^{6} \cdot$ Stephane Fournier $^{2}$ - Carlos Van Mieghem ${ }^{2} \cdot$ Martin Penicka $^{2}$ Martin Mates $^{7}$. Frank Van Praet $^{2}$. \\ Ivan Degriek $^{2}$ Emanuele Barbato ${ }^{2,8}$
}

Received: 14 April 2018 / Accepted: 2 July 2018 / Published online: 19 July 2018

(C) Springer Science+Business Media, LLC, part of Springer Nature 2018

\begin{abstract}
Clinical benefit of invasive functionally guided revascularization has been mostly investigated and proven for percutaneous coronary intervention. It has never been prospectively evaluated whether a systematic fractional flow reserve (FFR) assessment is also beneficial in guiding coronary artery bypass graft surgery (CABG). The objective of the GRAft patency after FFR-guided versus angiography-guIded CABG (GRAFFITI) trial was to compare an FFR-guided revascularization strategy to the traditional angiography-guided revascularization strategy for patients undergoing CABG. Patients were enrolled with significantly diseased left anterior descending or left main stem and at least one major coronary artery with angiographically intermediate stenosis (30-90\% diameter stenosis) that was assessed by FFR. Thereafter, while the FFR values were kept concealed, cardiac surgeons decided their intended procedural strategy based on the coronary angiography alone. At this point, patients underwent 1:1 randomization to either an FFR-guided or an angiography-guided CABG strategy. In case the patient was randomized to angiography-guided arm, cardiac surgeons kept their intended procedural strategy, i.e., CABG was guided solely on the basis of the coronary angiography. In case the patient was randomized to the FFR-guided arm, FFR values were disclosed to the surgeons who revised the surgical protocol according to the functional significance of each coronary stenosis. The primary endpoint of the trial was the rate of graft occlusion at 12 months, assessed by coronary computed tomography or coronary angiography. The secondary endpoints were (1) length of postoperative hospital stay; (2) changes in surgical strategy depending upon FFR results (in FFR-guided group only); and (3) rate of major adverse cardiac and cerebrovascular events, i.e., composite of death, myocardial infarction, stroke, and any revascularization during the follow-up period. This study is the first prospective randomized trial investigating potential clinical benefits, associated with FFR-guided surgical revascularization. Trial registration: NCT01810224
\end{abstract}

Keywords Coronary artery bypass graft surgery $\cdot$ Fractional flow reserve $\cdot$ Coronary angiography $\cdot$ Graft patency

\section{Abbreviations \\ CABG Coronary artery bypass graft surgery \\ CAD Coronary artery disease}

CCTA Coronary computed tomography angiography

FFR Fractional flow reserve

LAD Left anterior descending coronary artery
Associate Editor Enrique Lara-Pezzi oversaw the review of this article

Emanuele Barbato

emanuele.barbato@olvz-aalst.be

1 Department of Cardiology, University Heart Center Graz, Medical University of Graz, Graz, Austria

2 Cardiovascular Research Center Aalst, OLV Clinic, OLV Hospital, Moorselbaan, 164, B-9300 Aalst, Belgium

3 Department of Cardiology and Internal Medicine, University Hospital Brno and Medical Faculty of Masaryk University, Brno, Czech Republic
4 Division of Cardiology, Department of Medicine, University of Verona, Verona, Italy

5 Hospital Santa Marta - Centro Hospitalar Lisboa Central, Lisbon, Portugal

6 Hungarian Institute of Cardiology, Budapest, Hungary

7 Cardiovascular Center, Na Homolce Hospital, Prague, Czech Republic

8 Division of Cardiology, Department of Advanced Biomedical Sciences, Federico II University, Naples, Italy 
LM Left main coronary artery

PCI Percutaneous coronary intervention

\section{Introduction}

Several large registries have proven major discrepancy between the angiographic appearance and the functional importance of coronary stenoses, limiting the appropriate therapeutic decisionmaking when purely assessed by angiography [1-3]. Accordingly, the latest joined guidelines of the European Society of Cardiology and European Association of CardioThoracic Surgery $[4,5]$ on myocardial revascularization underscore the importance that myocardial revascularization in patients with stable coronary artery disease (CAD) should be justified with demonstrated significant myocardial ischemia. Consistently, revascularization of angiographically equivocal coronary stenoses should be limited to lesions responsible for reversible ischemia based on invasive fractional flow reserve (FFR) assessment. This recommendation is supported by the fact that percutaneous coronary intervention (PCI) of all and exclusively the lesions with FFR $\leq 0.80$ results into an improved clinical outcome overcoming the traditional way to assess the stenosis severity based mainly upon coronary angiography $[6,7]$.

However, it has never been prospectively evaluated whether a systematic FFR assessment is also beneficial as compared to the traditional angiographic evaluation in guiding coronary artery bypass graft surgery (CABG). The available data are in fact coming either from retrospective or from single-arm prospective registries. Botman et al. [8] found a significant relation between functional severity of CAD determined by FFR and graft patency at 1 year. Similarly, in a retrospective registry, when comparing FFR-guided and angiography-guided CABG, a significantly higher graft patency rate was observed in the FFR-guided group. However, no significant difference was observed in terms of major adverse clinical endpoints at 3 years [9]. These preliminary data advocated the need for a randomized clinical trial prospectively evaluating the potential benefit of FFR-guided over angiography-guided CABG.

The objective of the GRAft patency after FFR-guided versus angiography-guIded CABG (GRAFFITI) trial was to assess the importance of functional assessment of CAD prior to CABG. In particular, an FFR-guided strategy was compared to the traditional angiography-guided strategy in the procedural planning and performance of surgical revascularization. Based on previous data [8], we hypothesized that rate of graft patency in the case of FFR-guided bypass surgery is significantly higher at 1year follow-up, than in the case of angiography-guided bypass surgery. We also presumed that FFR-guidance might be beneficial in terms of recovery and length of hospitalization, mainly through a reduction in the need of more extensive surgical intervention (i.e., reduced number of grafts needed; reduced rate of on-pump surgical approach)[9].
Methods

\section{Patient Population}

From March 2012 to December 2016, all consecutive patients undergoing elective coronary angiography for stable angina, unstable angina or non-ST elevation acute coronary syndrome were screened for possible inclusion in the presence of (1) significant stenosis of the left main stem (LM) and/or the left anterior descending coronary artery (LAD) proven by angiography or by FFR $(\leq 0.80)$ and $(2)$ at least one other major coronary artery with a lesion of diameter stenosis between 30 and $90 \%$ by visual assessment $[4,5]$ in any of the other coronary arteries.

Patients were excluded in case of (1) acute ST elevation myocardial infarction; (2) moderate to severe valve disease with indication for valve surgery; (3) severe left ventricular dysfunction (ejection fraction $<35 \%$ ); and (4) atrial fibrillation, if MAZE procedure was considered.

As of March 2012, recruitment was slow with about 160 patients enrolled by December 2016; therefore, steering committee decided for closing enrollment.

\section{Study Design}

The GRAFFITI trial is designed as a single-blinded, openlabel, prospective 1:1 randomized controlled multi-center trial, conducted in the following 6 European centers: the Cardiovascular Research Center Aalst-OLV Hospital (Aalst, Belgium), the University of Verona (Verona, Italy), the University Hospital of Brno (Brno, Czech Republic), the Hospital Santa of Marta (Lisbon, Portugal), the Hungarian Institute of Cardiology (Budapest, Hungary), and the $\mathrm{Na}$ Homolce Hospital (Prague, Czech Republic). The trial is registered on www.clinicaltrials.gov (NCT01810224).

The study design is summarized in Fig. 1. Briefly, the study was designed to compare angiography-guided with FFRguided revascularization in patients undergoing coronary artery bypass surgery, based on significant LM or LAD disease $[4,5]$. Patients were enrolled after having signed the written informed consent and if they meet all the eligibility criteria, namely having a significantly diseased LAD or LM (by angiography or by FFR) and at least one more major coronary artery with an angiographically intermediate stenosis (3090\% diameter stenosis).

After enrollment, FFR was measured during the same procedure in all intermediate stenoses. Note that the FFR values were concealed and the cardiologist involved in the procedure did not participate further to discuss on the revascularization strategy for the patient. Thereafter, patients were discussed within the heart team and cardiac surgeons defined their intended surgical revascularization strategy based on the coronary angiography alone, blinded to the FFR values. In 
Fig. 1 GRAft patency after FFRguided versus angiographyguIded CABG TrIal (GRAFFITI) (LAD, left anterior descending coronary artery; LM, left main stem; FFR, fractional flow reserve; DS, diameter stenosis; CCTA, coronary computed tomography angiography; CA, coronary angiography)

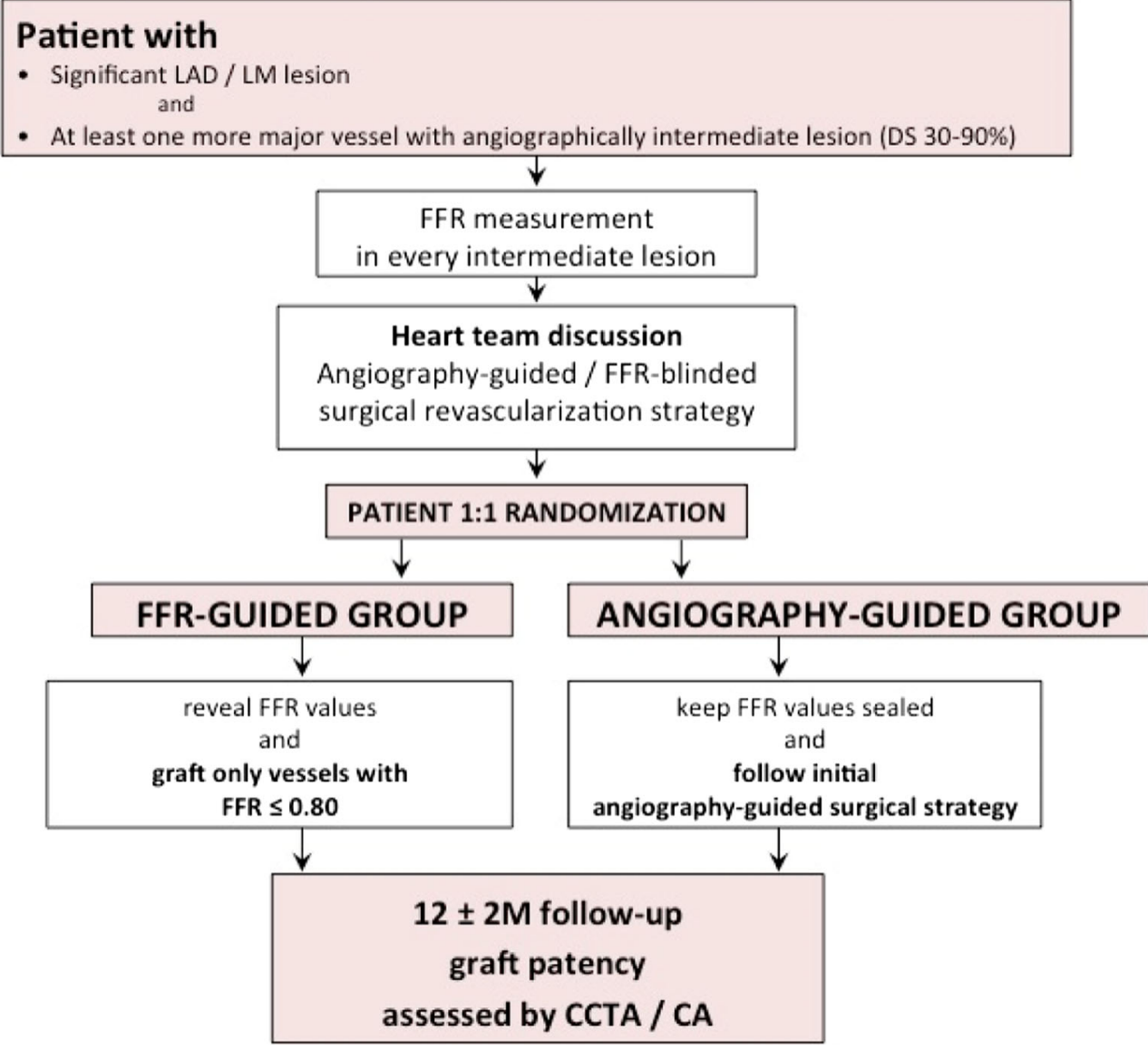

particular, they identified the number and type of grafts, the number of anastomoses and the surgical technique. The consensus on the surgical strategy was recorded in the case report form. At this point, patients underwent 1:1 randomization to either an FFR-guided or an angiography-guided CABG strategy. Randomization was done by a web-based platform. In case the patient was randomized to angiography-guided arm, FFR values remained concealed and cardiac surgeons performed the intended CABG strategy according to what predefined on the angiogram. In case the patient was randomized to the FFR-guided arm, measured FFR values were disclosed to the surgeons who were mandated to readjust the surgical protocol according to the functional significance of each coronary stenoses, regardless of the angiographic appearance, i.e., stenoses with FFR $\leq 0.80$ were grafted, while stenoses with FFR $>0.80$ were left alone. Changes in the revascularization strategies based upon functional information as compared with the initially intended strategy were recorded.

\section{Endpoints}

The primary endpoint of the trial was the rate of graft occlusion at 12 months. For evaluation, coronary computed tomography angiography (CCTA) was favored, considering its reliability in detecting graft patency [10]. Assessment was done by two experienced cardiologists (CVM, MP), blinded to randomization. All grafts were considered as patent or occluded. In case of clinically indicated coronary angiography, graft patency was assessed during the angiographic examination according to good clinical practice. In the latter case, CCTA was not performed.

The secondary endpoints were (1) length of postoperative hospital stay; (2) changes in surgical strategy depending upon FFR results (in FFR-guided group only), i.e., open-chest surgery vs. mini-thoracotomy; on-pump vs. off-pump; and number of grafts and anastomoses; and (3) rate of major adverse cardiac and cerebrovascular events, i.e., composite of death, myocardial infarction and stroke, and any revascularization during the follow-up period.

\section{Statistics}

Based on the limited data in the literature [8, 9], we assumed an absolute difference of $10 \%$ in the rate of occluded graft at 1 year between the two groups. Therefore, we calculated a sample size of 206 patients with $80 \%$ power, an alpha of 0.05 , and a $20 \%$ lost to follow-up.

Continuous variables will be compared by unpaired Student's $t$ tests, Mann-Whitney tests, or ANOVA test and categorical variables will be compared with Fisher's exact or 
chi-square tests, as appropriate. The difference in survival will be calculated by applying the Kaplan-Meier curves. Results will be adjusted by Cox-regression multivariate analysis, as appropriate. A probability value of $p<0.05$ is considered as significant.

\section{Discussion}

While there are already strong data supporting functionally guided percutaneous revascularization, data on functionally guided surgical revascularization is still limited in the literature. Accordingly, coronary artery bypass surgery is still following mainly the traditional way of angiogram-based revascularization.

Existing data so far are derived from observational studies and they seem to support the prognostic role of FFR in patients with planned CABG in terms of complexity of surgery, graft patency, etc. [11]. Still, since data from a prospective randomized clinical trial are still missing to confirm the concept, that FFR might play an important role in risk stratification and determining management strategy for these patients.

GRAFFITI trial is the first of its kind evaluating the use of FFR-guidance for patient assessment prior CABG. This study will provide novel insight on the potential impact of invasive functional guidance of $\mathrm{CABG}$ on surgical strategy, number of clinically indicated grafts and their long-term patency.

Based on a robust wealth of data, proving the clinical benefit of FFR-guided revascularization, the traditional anatomybased revascularization paradigm has been already shifted towards a more functionality-centered concept in the PCI field $[4-7,12,13]$. However, surgical revascularization is still mainly following the angiographic paradigm. This study might be the first prospective randomized trial, investigating potential clinical benefits, associated with FFR-guided surgical revascularization. Accordingly, results of the study might allow understanding, whether the same paradigm-shift is needed in surgical revascularization, as well.

Funding The GRAFFITI trial is an investigators' initiated trial supported by a limited unrestricted grant (less than 15.000 euros) of Abbott Vascular/St. Jude Medical (St. Paul, MN, US) used to support research nurses in data collection.

\section{Compliance with Ethical Standards}

This study is aligned with the Helsinki Declaration's principles, meaning all patients signed a written informed consent stating that participation was voluntary and that participation could be withdrawn at any time without any negative consequences concerning their current or future medical treatment. Study approval was obtained from the local ethical committees of each participating centers.

Conflict of Interest Drs. Barbato and De Bruyne report research grants and consulting fees to the Cardiovascular Research Institute Aalst
(Belgium) on their behalf from Abbott Vascular (St. Jude Medical). Dr. $\mathrm{G}$ Toth reports research grants and consulting fees from Abbott Vascular (St. Jude Medical), Medtronic, Boston Scientific and Biotronik. No other potential conflict of interest relevant to this original research was reported.

\section{References}

1. Tonino, P. A., Fearon, W. F., De Bruyne, B., Oldroyd, K. G., Leesar, M. A., Ver Lee, P. N., Maccarthy, P. A., Van't Veer, M., \& Pijls, N. H. (2010). Angiographic versus functional severity of coronary artery stenoses in the FAME study fractional flow reserve versus angiography in multivessel evaluation. Journal of the American College of Cardiology, 55, 2816-2821.

2. Hamilos, M., Muller, O., Cuisset, T., Ntalianis, A., Chlouverakis, G., Sarno, G., Nelis, O., Bartunek, J., Vanderheyden, M., Wyffels, E., Barbato, E., Heyndrickx, G. R., Wijns, W., \& De Bruyne, B. (2009). Long-term clinical outcome after fractional flow reserveguided treatment in patients with angiographically equivocal left main coronary artery stenosis. Circulation, 120, 1505-1512.

3. Toth, G., Hamilos, M., Pyxaras, S., Mangiacapra, F., Nelis, O., De Vroey, F., Di Serafino, L., Muller, O., Van Mieghem, C., Wyffels, E., Heyndrickx, G. R., Bartunek, J., Vanderheyden, M., Barbato, E., Wijns, W., \& De Bruyne, B. (2014). Evolving concepts of angiogram: fractional flow reserve discordances in 4000 coronary stenoses. European Heart Journal, 35, 2831-2838.

4. Task Force on Myocardial Revascularization of the European Society of Cardiology (ESC) and the European Association for Cardio-Thoracic Surgery (EACTS); European Association for Percutaneous Cardiovascular Interventions (EAPCI), Wijns, W., Kolh, P., Danchin, N., Di Mario, C., Falk, V., Folliguet, T., Garg, S., Huber, K., James, S., Knuuti, J., Lopez-Sendon, J., Marco, J., Menicanti, L., Ostojic, M., Piepoli, M. F., Pirlet, C., Pomar, J. L., Reifart, N., Ribichini, F. L., Schalij, M. J., Sergeant, P., Serruys, P. W., Silber, S., Sousa Uva, M., \& Taggart, D. (2010). Guidelines on myocardial revascularization. European Heart Journal, 31, 25012555.

5. Task Force on Myocardial Revascularization of the European Society of Cardiology (ESC) and the European Association for Cardio-Thoracic Surgery (EACTS); European Association for Percutaneous Cardiovascular Interventions (EAPCI), Kolh, P., Wijns, W., Danchin, N., Di Mario, C., Falk, V., Folliguet, T., Garg, S., Huber, K., James, S., Knuuti, J., Lopez-Sendon, J., Marco, J., Menicanti, L., Ostojic, M., Piepoli, M. F., Pirlet, C., Pomar, J. L., Reifart, N., Ribichini, F. L., Schalij, M. J., Sergeant, P., Serruys, P. W., Silber, S., Sousa Uva, M., \& Taggart, D. (2010). Guidelines on myocardial revascularization. European Journal of Cardio-Thoracic Surgery, 38(Suppl), S1-S52.

6. van Nunen, L. X., Zimmermann, F. M., Tonino, P. A., Barbato, E., Baumbach, A., Engstrøm, T., Klauss, V., MacCarthy, P. A., Manoharan, G., Oldroyd, K. G., Ver Lee, P. N., Van't Veer, M., Fearon, W. F., De Bruyne, B., Pijls, N. H., \& FAME Study Investigators. (2015). Fractional flow reserve versus angiography for guidance of PCI in patients with multivessel coronary artery disease (FAME): 5-year follow-up of a randomised controlled trial. Lancet, 386, 1853-1860.

7. Xaplanteris, P., Fournier, S., Pijls, N. H. J., Fearon, W. F., Barbato, E., Tonino, P. A. L., Engstrøm, T., Kääb, S., Dambrink, J. H., Rioufol, G., Toth, G. G., Piroth, Z., Witt, N., Fröbert, O., Kala, P., Linke, A., Jagic, N., Mates, M., Mavromatis, K., Samady, H., Irimpen, A., Oldroyd, K., Campo, G., Rothenbühler, M., Jüni, P., De Bruyne, B., \& FAME 2 Investigators. Five-year outcomes with PCI guided by fractional flow reserve. New England Journal of Medicine, 2018. https://doi.org/10.1056/NEJMoa1803538. 
8. Botman, C. J., Schonberger, J., Koolen, S., Penn, O., Botman, H., Dib, N., Eeckhout, E., \& Pijls, N. (2007). Does stenosis severity of native vessels influence bypass graft patency? A prospective fractional flow reserve-guided study. Annals of Thoracic Surgery, 83, 2093-2097.

9. Toth, G., De Bruyne, B., Casselman, F., De Vroey, F., Pyxaras, S., Di Serafino, L., Van Praet, F., Van Mieghem, C., Stockman, B., Wijns, W., Degrieck, I., \& Barbato, E. (2013). Fractional flow reserve-guided versus angiography-guided coronary artery bypass graft surgery. Circulation, 128, 1405-1411.

10. Weustink, A. C., Nieman, K., Pugliese, F., Mollet, N. R., Meijboom, W. B., van Mieghem, C., ten Kate, G. J., Cademartiri, F., Krestin, G. P., \& de Feyter, P. J. (2009). Diagnostic accuracy of computed tomography angiography in patients after bypass grafting: comparison with invasive coronary angiography. JACC Cardiovasc Imaging., 2, 816-824.

11. Pellicano, M., De Bruyne, B., Toth, G. G., Casselman, F., Wijns, W., \& Barbato, E. (2017). Fractional flow reserve to guide and to assess coronary artery bypass grafting. European Heart Journal, 38, 1959-1968.

12. Zimmermann, F. M., Ferrara, A., Johnson, N. P., van Nunen, L. X., Escaned, J., Albertsson, P., Erbel, R., Legrand, V., Gwon, H. C., Remkes, W. S., Stella, P. R., van Schaardenburgh, P., Bech, G. J., De Bruyne, B., \& Pijls, N. H. (2015). Deferral vs. performance of percutaneous coronary intervention of functionally non-significant coronary stenosis: 15-year follow-up of the DEFER trial. European Heart Journal, 36, 3182-3188.

13. De Bruyne, B., Fearon, W. F., Pijls, N. H., Barbato, E., Tonino, P., Piroth, Z., Jagic, N., Mobius-Winckler, S., Rioufol, G., Witt, N., Kala, P., MacCarthy, P., Engström, T., Oldroyd, K., Mavromatis, K., Manoharan, G., Verlee, P., Frobert, O., Curzen, N., Johnson, J. B., Limacher, A., Nüesch, E., Jüni, P., \& FAME 2 Trial Investigators. (2014). Fractional flow reserve-guided PCI for stable coronary artery disease. New England Journal of Medicine, 371, 1208-1217. 